\title{
Subcutaneous Panniculitis-Like T-Cell Lymphoma with Hemophagocytic Syndrome in a Child: A Successful Treatment with the BFM-NHL-90 Protocol
}

\author{
Sunjoo Kim ${ }^{1}$, Aeri Kim² and Jeong Ok Hah ${ }^{1}$ \\ Departments of ${ }^{1}$ Pediatrics and ${ }^{2}$ Pathology, Daegu Fatima Hospital, Daegu, Korea
}

Subcutaneous panniculitis-like T-cell lymphoma (SPTCL) is a distinct cutaneous lymphoma subtype that is characterized by pleomorphic T-cell infiltration of the subcutaneous tissue. SPTCL is usually associated with indolent clinical course. However, it can be complicated by hemophagocytic syndrome (HPS), which leads to worse prognosis. Childhood SPTCL is rare and there is no standardized treatment regimen of SPTCL with HPS. Here we report a pediatric case of SPTCL with HPS who responded favorably with multi-agent chemotherapy of the BFM (Berlin-Frankfurt-Münster)-NHL (non-Hodgkin lymphoma)-90 protocol.

Key Words: Subcutaneous panniculitis-like T-cell lymphoma, Hemophagocytic syndrome, BFM-NHL-90
pISSN 2233-5250 / elSSN 2233-4580 https://doi.org/10.15264/cpho.2020.27.2.129 Clin Pediatr Hematol Oncol 2020;27:129 133

Received on August 20, 2020 Revised on October 5, 2020 Accepted on October 14, 2020

Corresponding Author: Jeong Ok Hah Department of Pediatrics, Daegu Fatima Hospital, 99 Ayang-ro, Dong-gu, Daegu 41199, Korea Tel: $+82-53-940-7244$ Fax: +82-53-954-7417

E-mail: johah@med.yu.ac.kr ORCID ID: orcid.org/0000-0002-4072-7867

\section{Introduction}

Subcutaneous panniculitis-like T-cell lymphoma (SPTCL) is a distinct subtype of skin lymphoma that is characterized by subcutaneous tissue infiltration by pleomorphic $\mathrm{T}$ cells and benign macrophages that mimic lobular panniculitis [1].

The presence of accompanying hemophagocytic syndrome (HPS) is one of the most important prognostic factor for SPTCL, which implies poorer overall survival $[2,3]$. The clinical course of SPTCL associated with HPS is generally aggressive, and a delay in diagnosis or treatment may result in a fatal outcome [3].

Childhood SPTCL is rare, and only a few cases have been described in the literature so far [4]. The treatment options for SPTCL are not well defined, and multiple chemotherapy protocols have been used in various reports [2,3]. And there have been few reports of successful treatment of SPTCL with HPS [5].

Here, we report a pediatric case of SPTCL with HPS that was successfully treated with high-dose chemotherapy (HDT) of the BFM (Berlin-Frankfurt-Münster)-NHL (non-Hodgkin lymphoma)-90 protocol.

\section{Case Report}

A previously healthy 11-year-old boy was presented with multiple plaques and nodules in the inguinal area with intermittent fever, 2-kg weight loss, and general fatigue for the past 3 months.

The patient was evaluated elsewhere and underwent inguinal nodule biopsy. He was diagnosed as panniculitis and treated with low-dose oral corticosteroids and anti- 
biotics for a month. However, erythematous lesions on the groin gradually expanded with persistent fever.

He was therefore referred to our institute for further evaluation on February 2016. At the time of presentation to us, his body temperature was $38.5^{\circ} \mathrm{C}$ and there were several erythematous subcutaneous nodules with tenderness ranging from 2 to $4 \mathrm{~cm}$ in size affecting the axilla, groin and trunk (Fig. 1). Splenomegaly was also noted on physical examination.

Initial laboratory findings were as follows : WBC count

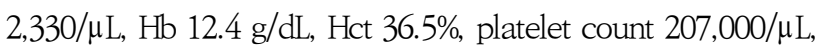
AST 75 IU/L (normal: 10-40), ALT 73 IU/L (normal: 5-35), lactic dehydrogenase (LDH) 858 IU/L (normal: 115-359).

Excisional biopsy of nodules on the groin was performed and the specimen showed subcutaneous infiltra- tion of atypical lymphocytes, rimming adipocytes (Fig. $2 \mathrm{~A})$. Immunohistochemistry on the tissue revealed positive for $\mathrm{CD} 3$ (Fig. 2B), CD5, and CD8 (Fig. 2C), and negative for CD4 (Fig. 2D), CD20, CD30, and CD56. T-cell receptor (TCR) gene rearrangement analysis for TCR- $\beta$ was positive. Bone marrow examination showed normocelluar marrow with no evidence of atypical lymphocyte infiltration or hemophagocytosis. An 18F-fluorodeoxyglucose (FDG) whole-body positron emission tomography (PET) scan revealed multiple increased FDG uptakes in the fat tissues of both supraclavicular areas, right axilla, both arms, trunk, subsplenic area, pelvis, both inguinal areas, genitalia, and left anterior thigh. It also showed increased FDG uptake in the nasopharynx and slightly increased FDG uptake in the spleen (Fig. 3A).
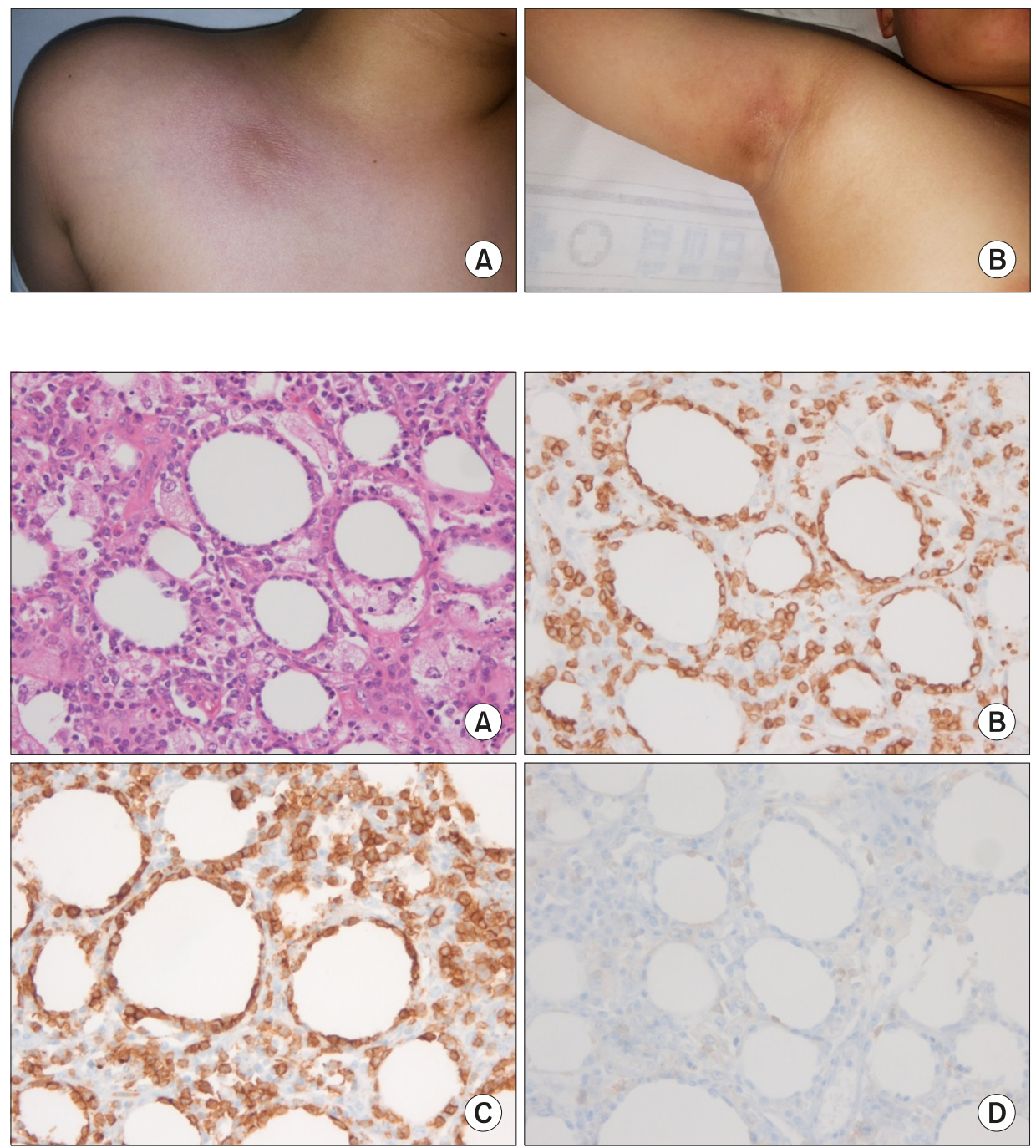

Fig. 1. Erythematous subcutaneous nodules on the anterior chest (A) and right axilla (B).

Fig. 2. Histopathologic findings of inguinal nodules. Hematoxylin-eosin stained specimen shows atypical lymphocytes infiltration with rimming adipocytes in the subcutaneous tissue (A). Immunohistostainings are positive for $\mathrm{CD} 3$ (B), CD8 (C) and, negative for CD4 (D) $(A-D$, original magnifications: $\times 200)$. 
A diagnosis of SPTCL was made based on histomorphology and T-cell immunophenotype. After the diagnosis of SPTCL with fever, leukopenia, splenomegaly and hepatitis, HPS was also suspicious. However, with the results of slightly elevated serum ferritin $[285.7 \mathrm{ng} / \mathrm{mL}$ (normal: 12-150)] and triglycerides [127 mg/dL (normal: 50-130)] within the normal range, the present case was suspected to have HPS which was not fully blown.

During the staging work-up, multiple nodules on the groin were considerably expanded and new lesions emerged on the both upper extremities within a day. Considering his aggressive and advanced features of SPTCL with suspicious HPS, although was not fully fulfilling the diag-

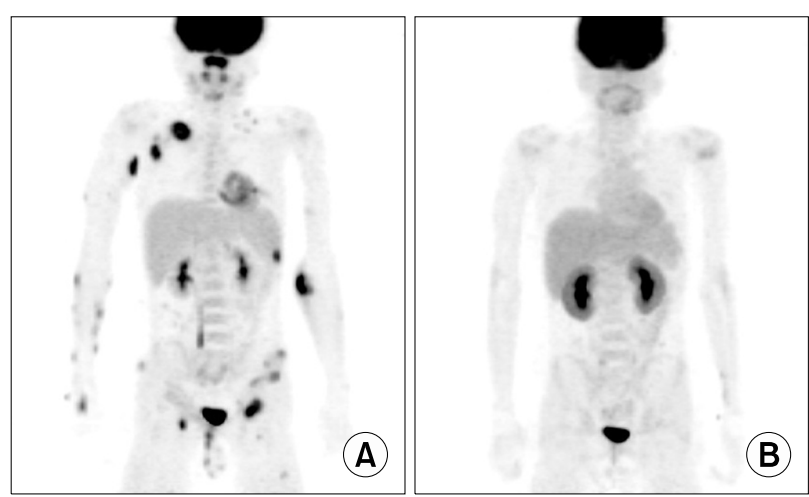

Fig. 3. (A) Whole-body PET scan at diagnosis reveals multiple increased FDG uptakes in the subcutaneous tissues of both arms, right axilla, trunk and groin. (B) Follow-up PET scan after the course AA-BB-CC of the BFM-NHL-90 protocol shows resolution of most of the lesions. nostic criteria of HPS, we initiated chemotherapy with the BFM-NHL-90 protocol, which is usually used in aggressive pediatric non-lymphoblastic lymphomas.

First cycle AA of the BFM-NHL-90 protocol was completed, and after several days of defervescence, he became febrile again with sustained splenomegaly. We repeated the laboratory tests and they revealed as fullblown HPS with the following findings: WBC count $2,420 / \mu \mathrm{L}$,

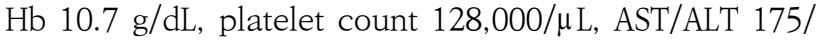
$310 \mathrm{IU} / \mathrm{L}$, LDH 858 IU/L, ferritin 620.6 ng/mL, triglyceride $836 \mathrm{mg} / \mathrm{dL}$. The serum soluble interleukin (IL)-2 receptor level was normal. Assessments for NK-cell activity and fibrinogen level were not performed.

As HPS was suspected to be secondary to SPTCL, and the BFM-NHL-90 protocol included dexamethasone and etoposide, which would help in controlling HPS as well, we continued BFM-NHL-90 protocol and added cyclosporine A (CsA) on the regimen. After course BB-CC, we conducted a follow-up PET scan and it showed resolution of the most of the lesions with no evidence of residual active lymphoma (Fig. 3B). He underwent four more cycles with regimen $\mathrm{A}-\mathrm{B}-\mathrm{A}-\mathrm{B}$. He suffered from methotrexate-induced oral mucositis and pneumonia, although chemotherapy with BFM-NHL-90 was otherwise well-tolerated.

The BFM-NHL-90 protocol was completed and he showed complete resolution of the skin lesions with nod-

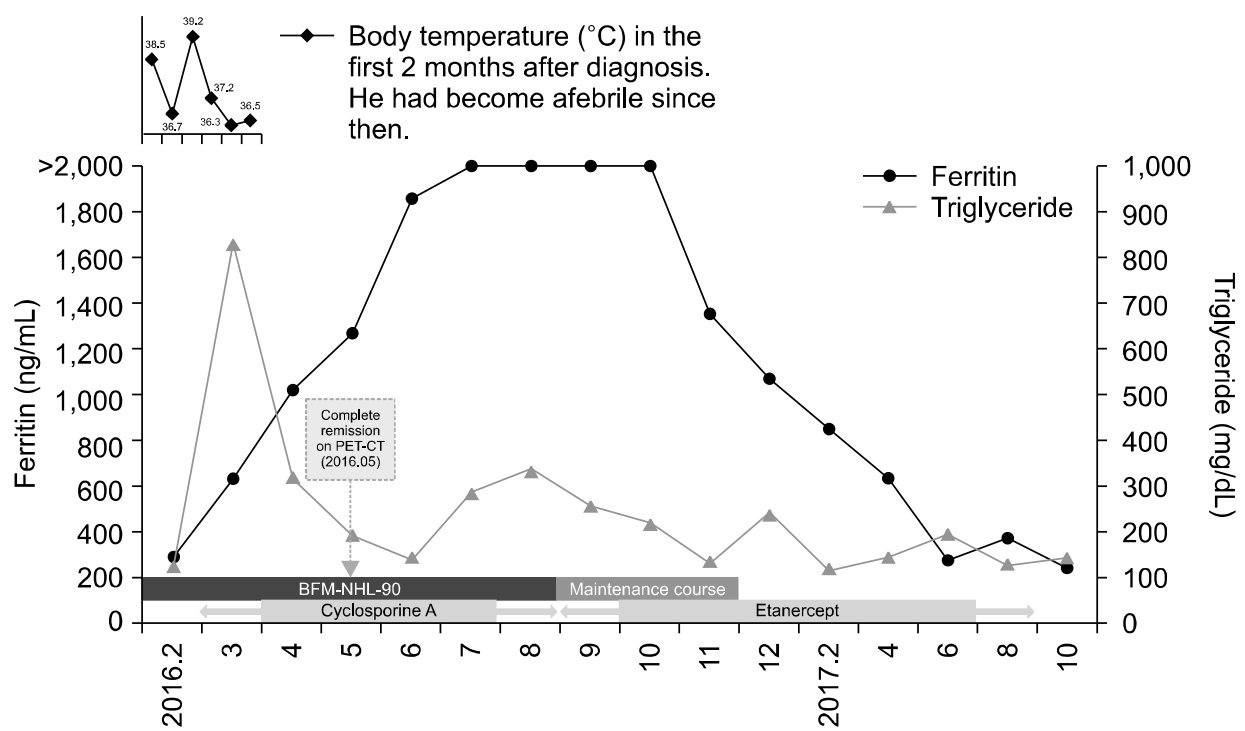

Fig. 4. Clinical course. Treatment regimens, body temperature and sequential changes of the levels of ferritin and triglyceride are shown. 
ules and features of HPS, except for persistent hyperferritinemia with a level of more than $2,000 \mathrm{ng} / \mathrm{mL}$. In view of sustained hyperferritinemia, as it might be due to the incomplete control of HPS, we planned to administer additional five cycles of maintenance therapy (every 2-weeks) with etoposide, vincristine, dexamethasone. With the discontinuation of CsA, etanercept, which is a tumor necrosis factor (TNF) $-\alpha$ inhibitor, was started $(25 \mathrm{mg} /$ dose, once a week) and continued for 1-year.

The highly elevated ferritin levels were gradually decreased (Fig. 4) and he has been in complete remission for more than 4 years.

\section{Discussion}

SPTCL is a distinct cutaneous lymphoma subtype characterized by the subcutaneous tissue infiltration by pleomorphic $\mathrm{T}$ cells rimming adipocytes, mimicking panniculitis [1]. On the basis of TCR gene phenotype, there are two subtypes of SPTCL: $\alpha / \beta$ and $\gamma / \delta$. In 2005, the World Health Organization (WHO) and European Organization for Research and Treatment of Cancer (EORTC) classification of primary cutaneous lymphomas have restricted the category of SPTCL to tumors expressing the TCR $\alpha / \beta$, placing those expressing the TCR $\gamma / \delta$ into a new provisional category of cutaneous $\gamma \delta$ T-cell lymphoma [6]. This division was based on the difference in prognoses between these two, with SPTCL showing much promising clinical outcomes [7].

The pediatric cases of SPTCL are rarely found and SPTCL is usually a disease of middle-aged adults [2]. SPTCL has generally been associated with indolent clinical course and favorable prognosis. However, the development of HPS is reported to occur in approximately $20 \%$ of SPTCL patients and is associated with a poorer outcome, with a 5-year survival of 46\% [3].

Although the mechanism of HPS in SPTCL has not been clarified, the phenomenon of HPS results from the overproduction of cytokines, including interferon- $\gamma$, IL-2, IL-6, IL-12, IL-18 and TNF- $\alpha$, produced by activated T cells (TH1 cells) and macrophages, which leads to a chain reaction of cytokines $[8,9]$. HPS is thought to be a re- active T-cell process mediated by cytokines in SPTCL because of which CsA, along with steroids, has also been used in this condition [10,11].

Controlling HPS might be the key treatment for SPTCL, with SPTCL having a high mortality rate despite the use of aggressive chemotherapy regimen. And the majority of fatal outcomes was a result of the complication of HPS and not to the lymphoma itself [12].

There were no standardized therapeutic approaches for the treatment of SPTCL with HPS, as treatments are seen to vary from corticosteroids, to multi-agent chemotherapy, and to stem cell transplantation (SCT) in various reports published to date $[2,3,5]$. Patients with systemic symptoms and involvement of multiple skin areas usually receive multi-agent chemotherapy. Suvir et al. reviewed seven cases of pediatric SPTCL with HPS [5]. Of the seven patients, three were treated with BFM-NHL-90, one with CHOP with allogeneic SCT, one with CHOP plus fludarabine, one with Bexarotene, prednisolone, SMILE (steroid, methotrexate, ifosfamide, L-asparaginase, and etoposide) and one with unknown treatment. All the three cases treated with BFM-NHL-90 achieved complete remission.

The present case showed aggressive features of SPTCL and accompanying HPS was suspected. We thus administered multi-agent chemotherapy with BFM-NHL-90 protocol and CsA was added later on. Cutaneous lesions showed complete resolution, and the majority of features of HPS were resolved after the HDT of the BFM-NHL-90 protocol with CsA; however, the highly elevated serum ferritin levels were sustained.

Although the pathophysiology of hyperferritinemia in HPS is not fully understood, Stroud J. hypothesized that macrophages can fuse with pathologic (infected or malignant) cells, and these hybrid cells "chew up" erythrocytes and secrete ferritin [13]. We speculated this hyperferritinemia might be owing to the insufficient control of HPS with the therapy we used. Therefore, CsA was discontinued and he was treated with etanercept along with maintenance therapy that included etoposide, dexamethasone and vincristine. Etanercept can be used in therapy-resistant macrophage activation syndrome (MAS)/ HPS and several successful treatment cases were reported 
$[14,15]$. After the adoption of the etanercept, the ferritin levels were gradually decreased. As etanercept did not show dramatic response on the hyperferritinemia, further study is warranted to determine etanercept could be an alternative approach for HPS with persistent hyperferritinemia.

In summary, we have reported a pediatric case of SPTCL complicated by HPS, which responded favorably to treatment with BFM-NHL-90 regimen. Although the value of the BFM-NHL-90 protocol has to be further evaluated in SPTCL with HPS cases, our findings suggest that the regimen may be applicable particularly in those with aggressive features.

\section{Conflict of Interest Statement}

The authors have no conflict of interest to declare.

\section{References}

1. Gonzalez CL, Medeiros LJ, Braziel RM, Jaffe ES. T-cell lymphoma involving subcutaneous tissue: a clinicopathologic entity commonly associated with hemophagocytic syndrome. Am J Surg Pathol 1991;15:17-27.

2. Go RS, Wester SM. Immunophenotypic and molecular features, clinical outcomes, treatments, and prognostic factors associated with subcutaneous panniculitis-like T-cell lymphoma. Cancer 2004;101:1404-13.

3. Willemze R, Jansen PM, Cerroni L, et al. Subcutaneous panniculitis-like T-cell lymphoma: definition, classification, and prognositc factors: an EORTC Cutaneous-Lymphoma Group Study of 83 cases. Blood 2008;111:838-45.

4. Huppmann AR, Xi L, Raffeld M, Pittaluga S, Jaffe ES. Subcuta- neous panniculitis-like T-cell lymphoma in the pediatric age group: a lymphoma of low malignant potential. Pediatr Blood Cancer 2013;60:1165-70.

5. Singh S, Philip CC, John MJ. Pediatric subcutaneous panniculitis-like T-cell lymphoma with hemophagocytosis showing complete resolution with the BFM90 protocol: case report and review of literature. J Pediatr Hematol Oncol 2019;41: 478-81.

6. Willemze R, Jaffe ES, Burg G, et al. WHO-EORTC classification for cutaneous lymphomas. Blood 2005;105:3768-85.

7. Toro JR, Liewehr DJ, Pabby N, et al. Gamma-delta T-cell phenotype is associated with significantly decreased survival in cutaneous T-cell lymphoma. Blood 2003;101:3407-12.

8. Akashi K, Hayashi S, Gondo H, et al. Involvement of interferon- $\gamma$ and macrophage colony-stimulating factor in pathogenesis of haemophagocytic lymphohistiocytosis in adults. $\mathrm{Br}$ J Haematol 1994;87:243-50.

9. Osugi Y, Hara J, Tagawa S, et al. Cytokine production regulating Th1 and Th2 cytokines in hemophagocytic lymphohistiocytosis. Blood 1997;89:4100-3.

10. Tsukamoto Y, Katsunobu Y, Omura Y, et al. Subcutaneous panniculitis-like T-cell lymphoma: successful initial treatment with prednisolone and cyclosporin A. Intern Med 2006; 45:21-4.

11. Shani-Adir A, Lucky AW, Prendiville J, et al. Subcutaneous panniculitic T-cell lymphoma in children: response to combination therapy with cyclosporine and chemotherapy. J Am Acad Dermatol 2004;50(Suppl 2):S18-22.

12. Wang CY, Su WP, Kurtin PJ. Subcutaneous panniculitic T-cell lymphoma. Int J Dermatol 1996;35:1-8.

13. Stroud J. A mechanistic theory explaining hyperferritinaemia in haemophagocytic lymphohistiocytosis. Med Hypotheses 2019; 122:165-71.

14. Takahashi N, Naniwa T, Banno S. Successful use of etanercept in the treatment of acute lupus hemophagocytic syndrome. Mod Rheumatol 2008;18:72-5.

15. Kikuchi H, Yamamoto T, Asako K, Takayama M, Shirasaki R, Ono Y. Etanercept for the treatment of intractable hemophagocytic syndrome with systemic lupus erythematosus. Mod Rheumatol 2012;22:308-11. 